\title{
Association of body composition with odds of breast cancer by molecular subtype: analysis of the Mechanisms for Established and Novel Risk Factors for Breast Cancer in Nigerian Women (MEND) study
}

Tomi Akinyemiju ${ }^{1,2,3^{*}}$, Kelley Jones ${ }^{1}$, Anjali Gupta ${ }^{1,4}$, Taofik Oyekunle ${ }^{1}$, Veeral Saraiya ${ }^{5}$, April Deveaux Omolola Salako ${ }^{6}$, Allison Hall ${ }^{7}$, Olusegun Alatise ${ }^{8}$, Gabriel Ogun ${ }^{9}$, Adewale Adeniyi ${ }^{10}$, Omobolaji Ayandipo ${ }^{9}$, Thomas Olajide ${ }^{6}$, Olalekan Olasehinde ${ }^{8}$, Olukayode Arowolo ${ }^{8}$, Adewale Adisa ${ }^{8}$, Oludolapo Afuwape ${ }^{9}$, Aralola Olusanya ${ }^{9}$, Aderemi Adegoke ${ }^{11}$, Trygve O. Tollefsbol ${ }^{12}$, Donna Arnett ${ }^{13}$, H3 Africa Kidney Research Network 14 and Adetola Daramola ${ }^{6}$

\begin{abstract}
Background: The association between obesity and breast cancer (BC) has been extensively studied among US, European and Asian study populations, with often conflicting evidence. However, despite the increasing prevalence of obesity and associated conditions in Africa, the continent with the highest age-standardized BC mortality rate globally, few studies have evaluated this association, and none has examined in relation to molecular subtypes among African women. The current analysis examines the association between body composition, defined by body mass index (BMI), height, and weight, and BC by molecular subtype among African women.

Methods: We estimated odds ratios (ORs) and 95\% confidence intervals (95\% Cl) for the association between measures of body composition and BC and molecular subtypes among 419 histologically confirmed cases of BC and 286 healthy controls from the Mechanisms for Established and Novel Risk Factors for Breast Cancer in Women of Nigerian Descent (MEND) case-control study.
\end{abstract}

\footnotetext{
* Correspondence: tomi.akinyemiju@duke.edu

'Department of Population Health Sciences, School of Medicine, Duke University, Durham, NC, USA

${ }^{2}$ Duke Cancer Institute, School of Medicine, Duke University, Durham, NC, USA

Full list of author information is available at the end of the article
}

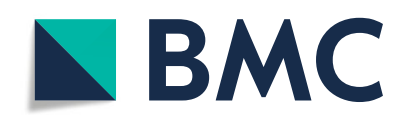

(- The Author(s). 2021 Open Access This article is licensed under a Creative Commons Attribution 4.0 International License, which permits use, sharing, adaptation, distribution and reproduction in any medium or format, as long as you give appropriate credit to the original author(s) and the source, provide a link to the Creative Commons licence, and indicate if changes were made. The images or other third party material in this article are included in the article's Creative Commons licence, unless indicated otherwise in a credit line to the material. If material is not included in the article's Creative Commons licence and your intended use is not permitted by statutory regulation or exceeds the permitted use, you will need to obtain permission directly from the copyright holder. To view a copy of this licence, visit http://creativecommons.org/licenses/by/4.0/ The Creative Commons Public Domain Dedication waiver (http://creativecommons.org/publicdomain/zero/1.0/) applies to the data made available in this article, unless otherwise stated in a credit line to the data. 
Results: Higher BMI (aOR: 0.79; 95\% Cl: 0.67, 0.95) and weight (aOR: 0.83; 95\% Cl: 0.69, 0.98) were associated with reduced odds of $B C$ in adjusted models, while height was associated with non-statistically significant increased odds of BC (aOR: 1.07, 95\% Cl: 0.90, 1.28). In pre/peri-menopausal, but not post-menopausal women, both higher $\mathrm{BMI}$ and weight were significantly associated with reduced odds of BC. Further, higher BMI was associated with reduced odds of Luminal A, Luminal B, and HER2-enriched BC among pre/peri-menopausal women, and reduced odds of triple-negative BC among post-menopausal women.

Conclusions: Higher BMI and weight were associated with reduced odds of BC overall and by molecular subtype among West African women. Larger studies of women of African descent are needed to definitively characterize these associations and inform cancer prevention strategies.

Keywords: Body composition, BMl, Breast cancer, Nigeria, Molecular subtype, Hormone receptor

\section{Background}

Breast cancer (BC) poses a significant global challenge, with an estimated 2.1 million new cases and over 0.6 million deaths occurring in 2018 [1]. The African continent has the highest age-standardized $\mathrm{BC}$ mortality rate globally, with Nigeria, the most populous African nation, experiencing the highest rate within the continent [2]. In addition, the past few decades have been characterized by rapid increases in $\mathrm{BC}$ incidence rates among African women [3]. This increase is thought to be partly attributable to an epidemiological transition-a trend characterized by a decline in infectious diseases and an increase in non-communicable diseases, including obesity, obesity-associated conditions, as well as cancer [4, 5]. Women of African descent are also more likely to experience disproportionately high rates of aggressive $\mathrm{BC}$ tumors with poorer prognosis [6-8]. Specifically, women of African descent, including African-American women in the United States [9] and Nigerian women, are more likely to be diagnosed with $\mathrm{BC}$ over-represented by the triple-negative (TN) subtypes, characterized by estrogen (ER), progesterone (PR), and human epidermal growth factor-2 (HER2) receptor negativity [7, 8].

Non-communicable disease risk factors, such as high body mass index (BMI), have been shown to influence $\mathrm{BC}$ risk. Several studies have found that higher BMI, an indicator of excess adiposity, is associated with increased risk of post-menopausal $\mathrm{BC}$, but reduced risk of premenopausal BC $[10,11]$. This association has been observed among diverse populations, including European [12], Latin American [13], and Asian [14] women. However, the few studies in Nigeria, where obesity rates are rapidly increasing [15], are mixed. Two studies have noted no significant associations between $\mathrm{BMI}$ and $\mathrm{BC}$ risk [16, 17], while studies assessing other measures of excess adiposity, such as waist circumference and waistto-hip ratio, observed an association with increased risk of BC in Nigerian women $[17,18]$. These measures of excess adiposity have been associated with metabolic dysregulation, including insulin resistance and hyperlipidemia [19], which have separately been shown to increase BC risk [20]. Additionally, studies considering body height have found a positive association between height and $\mathrm{BC}$ risk $[21,22]$, including two studies among Nigerian women [16, 23]. Molecular BC subtype has been shown to modify the association between excess adiposity and $\mathrm{BC}$ risk. Obese women were found to have a higher risk of $\mathrm{TN}$ and Luminal $\mathrm{A}$ BC than normal-weight women, whereas normal-weight women were more likely to present with HER2+ BC in a population from the United States [24]. Another United States study found that elevated waist-to-hip ratio was associated with increased risk of Luminal A BC among postmenopausal women, and increased risk of basal-like (TN) BC in both pre- and post-menopausal women [25]. However, no study to date has examined the link between BMI and BC molecular subtypes in West African women.

Improved understanding of the impact of obesity on $\mathrm{BC}$ and molecular subtypes in West African women will add to the growing literature that can help inform locally relevant $\mathrm{BC}$ prevention strategies and contribute to enhancing existing risk prediction and prognostic models. Findings will also contribute to improved understanding of the biological mechanisms underlying aggressive tumor subtypes in women of African descent. The purpose of the current study was to evaluate the association between BMI, height, and weight with odds of BC among West African women, and to examine differences across molecular subtypes.

\section{Methods \\ Study design}

The Mechanisms for Established and Novel Risk Factors for Breast Cancer in Women of Nigerian Descent (MEND) study has been previously described in detail [26]. Briefly, newly diagnosed BC patients from four tertiary hospitals in southwestern Nigeria were recruited into the research study. A trained research nurse at each site introduced the study and explained study 
requirements in detail to potential participants during clinical visits, and interested participants were assessed for eligibility. Exclusion criteria included other medical conditions that could interfere with participation in the study, mental impairment, or not being able to communicate in English themselves or through a family member to complete the survey. Written and verbal informed consent was obtained from all study participants. A comprehensive study questionnaire was administered to obtain information on sociodemographic characteristics, reproductive history, and self and family history of cancer. Anthropometric measurements were obtained by the research nurse, followed by blood sample collection, and tumor and adjacent normal tissue biopsy sample collection. All biospecimen were collected prior to any chemotherapy or surgical treatment. Tissue samples were processed at the clinic and stored in $-80^{\circ} \mathrm{C}$ freezers until shipment to the United States for further analysis. Participants received a N500 telephone recharge card (approximately US \$1.50) as well as supplies needed for their clinical biopsy (biopsy needle and ancillary items). Data on healthy controls without BC were obtained from the Human Heredity and Health Africa (H3A) Chronic Kidney Disease (CKD) case-control study; methodology for the H3A CKD study has been described elsewhere [27]. Briefly, H3A recruitment occurred between 2015 and 2017, overlapping with case recruitment and included the recruitment of healthy, community-based adult women in southwestern Nigeria and Ghana. Extensive socio-demographic, clinical, family history and behavioral risk factor data was collected from all control participants; those with history of cancer or missing data on cancer history were excluded from analysis. Overall, 419 BC cases and 286 healthy controls were included in the current analysis (Fig. 1). All study procedures were approved by Duke University and the participating hospitals' Institutional Review Boards.

\section{Breast cancer cases and subtyping}

$\mathrm{BC}$ diagnosis was confirmed in one of two ways: 1) pathology reports based on clinical tumor biopsy samples from the diagnosing hospital in Nigeria and reviewed by a trained pathologist, or 2) research tumor biopsy samples collected at recruitment (same time as clinical biopsy) and shipped to the United States for review by a pathologist. Participants were considered a confirmed cancer case if either report indicated a cancer diagnosis. Confirmed $\mathrm{BC}$ tumor samples were subjected to immunohistochemistry (IHC) either at the diagnosing hospital

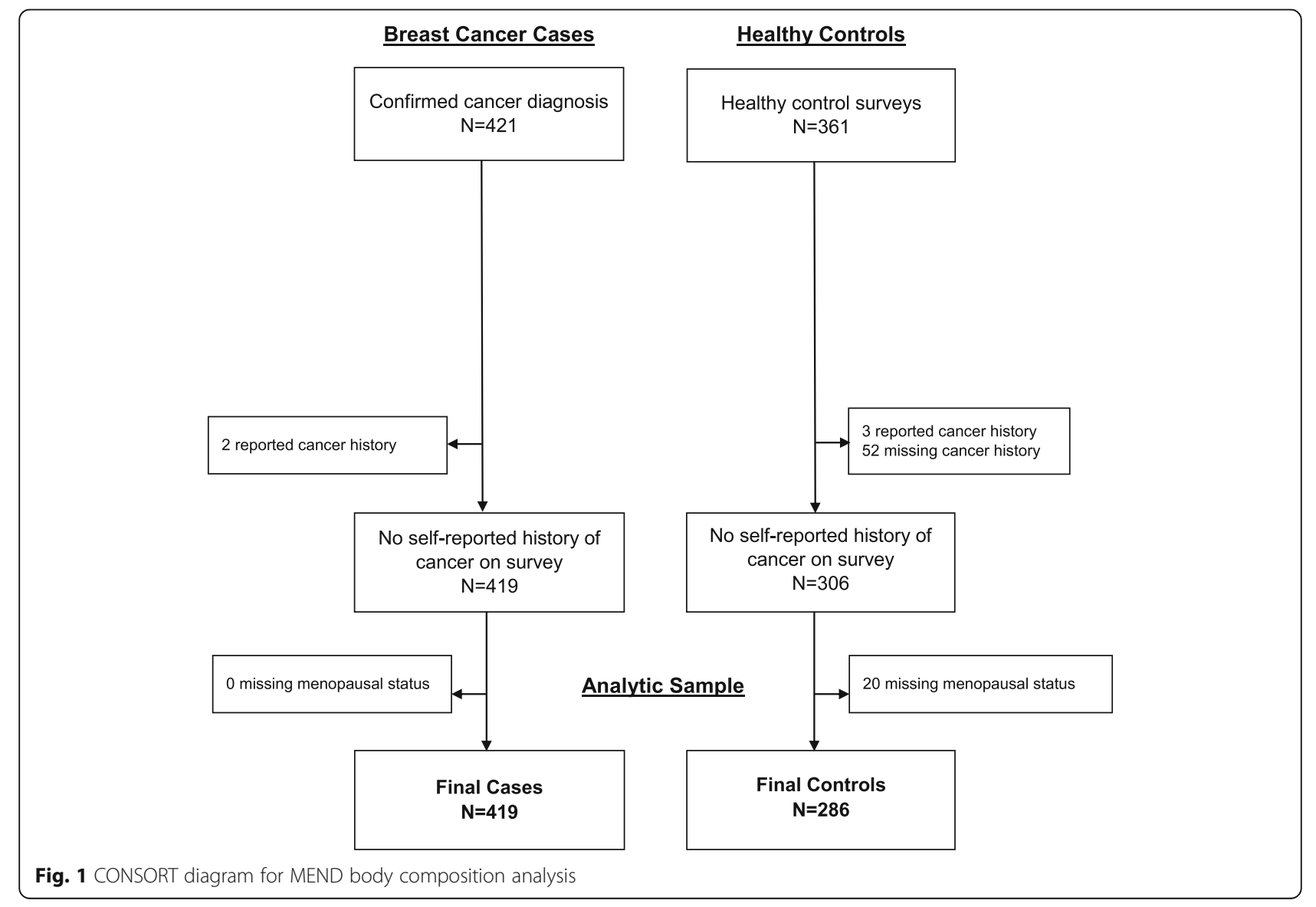


in Nigeria following standard protocol, or at the Duke University BioRepository and Precision Pathology Center. When both Nigeria and Duke IHC results were available, the Duke typing was used because it constituted the majority of IHC data on cases. Estrogen receptor and progesterone receptor status were scored using the Allred method [28, 29]. The proportion of nuclear positivity was scored as $0(0 \%), 1(<1 \%), 2(1-10 \%), 3$ $(11-33 \%), 4(34-66 \%)$ or $5(67-100 \%)$; intensity of the staining was scored as 0 (none), 1 (mild), 2 (moderate), or 3 (strong). These two scores were summed into positive (3-8) or negative (0-2). HER2 was scored as negative (scores 0,1$)$, equivocal $($ score $=2)$, positive $($ score $=$ 3) [30]. Cancer subtype was then classified as Luminal A (ER+ and/or PR+ / HER2-), Luminal B (ER+ and/or PR+ / HER2+), TN (ER-/PR-/HER2-), or HER2 (ER-/PR -/HER2+). In total, 169 cases had available data on ER/ PR/HER2 status for molecular subtype classification.

\section{Measures}

Anthropometric measurements for cases and controls were collected by trained research staff at enrollment, and included height, weight, and blood pressure. BMI was calculated from height and weight as $\mathrm{kg} / \mathrm{m}^{2}$. BMI, height, and weight were categorized into quartiles as well as continuous standardized variables by subtracting the sample mean and dividing by the sample standard deviation (SD). Quartiles were used rather than World Health Organization BMI categories $\left(<18.5 \mathrm{~kg} / \mathrm{m}^{2}\right.$ underweight, $18.5-<25$ normal weight, $25-<30$ overweight, and $30+$ obese) because it has been documented that these categories may not accurately capture risk in populations of African descent [31]. Reproductive and clinical history were self-reported by participants, and study covariates included age at menarche, parity, gravidity, menopausal status, and prior hypertension diagnosis. Participants also self-reported whether they had a history of other types of cancer; those with a positive history of cancer ( $<1 \%$ of cases and controls) or missing personal cancer history data (15\% of controls) were excluded from the analysis. Missing data for all measures were tabulated by case/control status. Variables with < $10 \%$ missing for both cases and controls were replaced with the median or modal value of the cases or controls, respectively, while variables with $>10 \%$ missing were not imputed.

\section{Analytical approach}

Descriptive statistics of proportions and frequencies for categorical variables and means $(\mathrm{SD})$ or medians (first quartile, third quartile) for continuous variables were used to characterize the sample by case/control status and BMI quartile. Differences in characteristics were tested using $X^{2}$ tests for categorical variables and
Wilcoxon rank-sum or Kruskal-Wallis nonparametric tests for continuous variables. The distribution of BMI was evaluated by case/control status and stratified by menopausal status and age group, respectively. The associations between body composition measures (BMI, height, and weight) and $\mathrm{BC}$ were tested using logistic regression models. Each body composition measure was assessed separately in a series of models: unadjusted, adjusted for age only, and adjusted for age, age at menarche, number of pregnancies, number of births, menopausal status, and prior hypertension diagnosis. A final model adjusted for BMI, height, and weight simultaneously in addition to all previous covariates. The primary models assessed quartiles as a categorical variable, with the lowest quartile set as the reference group. To test for trend across quartiles, the main exposure was analyzed as a continuous variable [1-4]. Finally, the standardized values for BMI, height, and weight were examined to determine the association per unit standard deviation (SD) increase in the measure with the odds of being a cancer case. These models were repeated with stratification for menopausal status. The subset of cases with molecular subtyping was analyzed using multinomial logistic regression models, specifying control status as the outcome reference group. The previous models were repeated to assess the odds of Luminal A, Luminal B, TN, or HER2 cancer subtypes. We assessed whether H3A control participants recruited from Nigeria and Ghana were significantly different in relation to key body composition measures; there were no significant difference between the groups, and in sensitivity analysis examining the analytical models using Nigerian controls only, results from the overall analysis were consistent, therefore analysis with the entire cohort of controls is presented. SAS v9.4 (SAS Institute, Cary, NC) was used for all analyses and significance was set at $\alpha=0.05$.

\section{Results}

A total of $419 \mathrm{BC}$ cases and 286 healthy controls were included in the analysis (Fig. 1). Median age at diagnosis or enrollment (48 years vs. 47 years), median age at menarche ( 15 years vs. 15 years), number of pregnancies ( 5 vs. 4) and number of births (4 vs. 4) was similar among cases and controls (Table 1). However, cases were less likely to report any prior use of hormone replacement therapy compared with controls. Approximately half (54\%) of cases and $60 \%$ of controls were overweight or obese, and controls were more likely to report a prior diagnosis of diabetes or hypertension. Among cases, the most common subtype was triple-negative (31\%), and $38 \%$ of cases were diagnosed at advanced grade (grade $3)$. Across BMI quartiles (Table 2), those in the highest quartile were older $(p<0.001)$, more likely to have a prior diagnosis of hypertension $(p<0.001)$, report ever 
Table 1 Clinical and reproductive characteristics among breast cancer cases and controls

\begin{tabular}{|c|c|c|}
\hline Study characteristics & $\begin{array}{l}\text { Case } \\
N=419\end{array}$ & $\begin{array}{l}\text { Control } \\
N=286\end{array}$ \\
\hline \multicolumn{3}{|l|}{ Demographics } \\
\hline Age $(\text { years })^{a}$ & $48.0(42.0,58.0)$ & $47.0(40.0,58.0)$ \\
\hline \multicolumn{3}{|l|}{ Clinical characteristics } \\
\hline \multicolumn{3}{|l|}{ BMI category } \\
\hline Underweight $(<18.5)$ & $21(5.0 \%)$ & $8(2.8 \%)$ \\
\hline Normal weight $(18.5-<25)$ & $172(41.1 \%)$ & $106(37.1 \%)$ \\
\hline Overweight $(25-<30)$ & $136(32.5 \%)$ & $94(32.9 \%)$ \\
\hline Obese $(30+)$ & $90(21.5 \%)$ & $78(27.3 \%)$ \\
\hline \multicolumn{3}{|l|}{ BMI quartile } \\
\hline$\leq 22.5$ & $117(27.9 \%)$ & $60(21.0 \%)$ \\
\hline$>22.5-\leq 25.6$ & $110(26.3 \%)$ & $71(24.8 \%)$ \\
\hline$>25.6-\leq 29.8$ & $99(23.6 \%)$ & $75(26.2 \%)$ \\
\hline$>29.8$ & $93(22.2 \%)$ & $80(28.0 \%)$ \\
\hline Height, $\mathrm{cm}^{\text {a }}$ & $160.0(156.0,164.1)$ & $159.7(155.0,163.5)$ \\
\hline Weight, $\mathrm{kg}^{\mathrm{a}}$ & $64.9(55.3,75.8)$ & $66.0(58.0,78.0)$ \\
\hline High blood pressure at enrollment & $134(32.0 \%)$ & $95(33.2 \%)$ \\
\hline Systolic BP a & $126.0(114.3,142.3)$ & $128.5(115.3,144.0$ \\
\hline Diastolic BP a & $80.0(70.7,88.7)$ & $77.0(70.0,87.7)$ \\
\hline \multicolumn{3}{|l|}{ Prior diabetes diagnosis } \\
\hline Yes & $6(1.4)$ & $43(15.0)$ \\
\hline No & $411(98.1)$ & $190(66.4)$ \\
\hline Missing & $2(0.5)$ & $53(18.5)$ \\
\hline Prior hypertension diagnosis & $82(19.6 \%)$ & $134(46.9 \%)$ \\
\hline \multicolumn{3}{|l|}{ Reproductive history } \\
\hline Age at menarche ${ }^{a}$ & $15.0(14.0,16.0)$ & $15.0(14.0,17.0)$ \\
\hline Ever pregnant & $399(95.2 \%)$ & $269(94.1 \%)$ \\
\hline Number of pregnancies $a, b$ & $5.0(3.0,6.0)$ & $4.0(3.0,6.0)$ \\
\hline Number of births ${ }^{a, b}$ & $4.0(3.0,5.0)$ & $4.0(2.0,5.0)$ \\
\hline \multicolumn{3}{|l|}{ Menopausal status } \\
\hline Pre- or peri-menopause & 207 (49.4\%) & $133(46.5 \%)$ \\
\hline Post-menopause & $212(50.6 \%)$ & $153(53.5 \%)$ \\
\hline \multicolumn{3}{|l|}{ Ever used HRT } \\
\hline Yes & $3(0.7)$ & $41(14.3)$ \\
\hline No & $416(99.3)$ & $189(66.1)$ \\
\hline Missing & $0(0.0)$ & $56(19.6)$ \\
\hline Cancer type & $(n=169)$ & $\mathrm{n} / \mathrm{a}^{\mathrm{c}}$ \\
\hline Luminal A & $46(27.2 \%)$ & \\
\hline Luminal B & $34(20.1 \%)$ & \\
\hline Triple negative & $52(30.8 \%)$ & \\
\hline HER2 & $37(21.9 \%)$ & \\
\hline Grade & & $\mathrm{n} / \mathrm{a}^{\mathrm{c}}$ \\
\hline 1 & $6(1.4 \%)$ & \\
\hline 2 & $132(31.5 \%)$ & \\
\hline
\end{tabular}


Table 1 Clinical and reproductive characteristics among breast cancer cases and controls (Continued)

\begin{tabular}{lll}
\hline Study characteristics & Case & Control \\
& $\mathbf{N = 4 1 9}$ & $\mathbf{N = \mathbf { 2 8 6 }}$ \\
\hline 3 & $84(20.1 \%)$ & \\
Unknown/Missing & $197(47.0 \%)$ & $\mathrm{n} / \mathrm{a}^{\mathrm{d}}$ \\
Prior Mammography Screenings & $78(18.6 \%)$ & $\mathrm{n} / \mathrm{a}^{\mathrm{d}}$ \\
Any Family History of Cancer & $21(5.0 \%)$ & \\
\hline
\end{tabular}

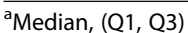

${ }^{\mathrm{b}}$ Among those who were ever pregnant

${ }^{c}$ Cancer variables are not applicable to control participants

dUnavailable for control participants

Table 2 Clinical and reproductive characteristics by BMl quartile among breast cancer cases and controls

\begin{tabular}{|c|c|c|c|c|c|}
\hline Study characteristics & $\begin{array}{l}\text { 1st quartile: } \\
\text { BMI } \leq 22.5 \\
N=177\end{array}$ & $\begin{array}{l}\text { 2nd quartile: } \\
\text { BMI }>22.5-\leq 25.6 \\
N=181\end{array}$ & $\begin{array}{l}\text { 3rd quartile: } \\
\text { BMI > 25.6 }-\leq 29.8 \\
N=174\end{array}$ & $\begin{array}{l}\text { 4th quartile: } \\
\text { BMI > 29.8 } \\
N=173\end{array}$ & $P$-value \\
\hline Case status & & & & & .10 \\
\hline Case & $117(66.1 \%)$ & $110(60.8 \%)$ & $99(56.9 \%)$ & $93(53.8 \%)$ & \\
\hline Control & $60(33.9 \%)$ & $71(39.2 \%)$ & $75(43.1 \%)$ & $80(46.2 \%)$ & \\
\hline \multicolumn{6}{|l|}{ Demographics } \\
\hline Age $\left(\right.$ years) ${ }^{a}$ & $46.0(37.0,55.0)$ & $48.0(40.0,58.0)$ & $47.5(41.0,56.0)$ & $52.0(44.0,59.0)$ & $<.001$ \\
\hline \multicolumn{6}{|l|}{ Clinical characteristics } \\
\hline Height, $\mathrm{cm}^{\mathrm{a}}$ & $161.0(156.0,164.5)$ & $160.0(156.0,164.0)$ & $160.0(155.0,163.8)$ & $160.0(156.0,164.1)$ & 69 \\
\hline Weight, $\mathrm{kg}^{\mathrm{a}}$ & $52.0(48.5,55.0)$ & $62.0(58.0,64.9)$ & $70.9(66.0,74.8)$ & $85.9(80.0,94.0)$ & $<.001$ \\
\hline High blood pressure at enrollment & $32(18.1)$ & $68(37.6)$ & $65(37.4)$ & $64(37.0)$ & $<.001$ \\
\hline Systolic BP a & $121.7(109.3,132.0)$ & $127.0(114.7,145.7)$ & $129.0(116.7,147.7)$ & $132.0(119.7,148.7)$ & $<.001$ \\
\hline Diastolic BP ${ }^{\mathrm{a}}$ & $76.0(66.7,84.0)$ & $78.3(68.7,89.3)$ & $79.2(72.3,88.3)$ & $80.3(74.7,89.7)$ & $<.001$ \\
\hline Prior diabetes diagnosis & & & & & .21 \\
\hline Yes & $7(4.0 \%)$ & $15(8.3 \%)$ & $11(6.3 \%)$ & $16(9.3 \%)$ & \\
\hline No & $159(89.8 \%)$ & $156(86.2 \%)$ & $143(82.2 \%)$ & $143(82.7 \%)$ & \\
\hline Missing & $11(6.2 \%)$ & $10(5.5 \%)$ & $20(11.5 \%)$ & $14(8.1 \%)$ & \\
\hline Prior hypertension diagnosis & $32(21.6 \%)$ & $41(28.5 \%)$ & $55(37.2 \%)$ & $65(48.1 \%)$ & $<.001$ \\
\hline \multicolumn{6}{|l|}{ Reproductive history } \\
\hline Age at menarche ${ }^{a}$ & $15.0(14.0,16.0)$ & $15.0(14.0,16.0)$ & $15.0(14.0,16.0)$ & $15.0(14.0,17.0)$ & .54 \\
\hline Ever pregnant & $161(91.0 \%)$ & $170(93.9)$ & $166(95.4)$ & $171(98.8)$ & .01 \\
\hline Number of pregnancies ${ }^{a, b}$ & $4.0(3.0,6.0)$ & $4.0(3.0,6.0)$ & $4.0(3.0,6.0)$ & $5.0(4.0,6.0)$ & .15 \\
\hline Number of births ${ }^{a, b}$ & $3.0(2.0,5.0)$ & $4.0(3.0,5.0)$ & $4.0(2.0,5.0)$ & $4.0(3.0,5.0)$ & .13 \\
\hline Menopausal status & & & & & .06 \\
\hline Pre- or peri-menopause & 97 (54.8\%) & $87(48.1 \%)$ & $86(49.4 \%)$ & $70(40.5 \%)$ & \\
\hline Post-menopause & $80(45.2 \%)$ & $94(51.9 \%)$ & $88(50.6 \%)$ & $103(59.5 \%)$ & \\
\hline Ever used HRT & & & & & .009 \\
\hline Yes & $4(2.3 \%)$ & $10(5.5 \%)$ & $11(6.3 \%)$ & $19(11.0 \%)$ & \\
\hline No & $159(89.8 \%)$ & $153(84.5 \%)$ & $152(87.4 \%)$ & $141(81.5 \%)$ & \\
\hline Missing & $14(7.9 \%)$ & $18(9.9 \%)$ & $11(6.3 \%)$ & $13(7.5 \%)$ & \\
\hline
\end{tabular}

Where applicable, missing values were not used to generate the $p$-value ${ }^{a}$ Median, (Q1, Q3)

${ }^{\mathrm{b}}$ Among those who were ever pregnant 
use of HRT ( $p=0.009)$ and be ever pregnant $(p=0.01)$. Furthermore, the distribution of BMI by case/control status stratified by menopausal status and age group (Fig. 2) indicated that the proportion of cases compared to controls in the lowest BMI quartile was higher across categories of menopausal status and age.

In multivariable regression models (Table 3), BMI in the highest versus lowest quartile (BMI $>29.8 \mathrm{~kg} / \mathrm{m}^{2}$ vs. $<22.5 \mathrm{~kg} / \mathrm{m}^{2}$ ) was associated with reduced odds of $B C$ in adjusted models (aOR: 0.56; 95\% CI: 0.33, 0.92; p-trend: 0.02). However, after additionally adjusting for height and weight, the estimate was no longer statistically significant (aOR: 0.32, 95\% CI: 0.10, 1.09; p-trend 0.06). Each SD increase in BMI was significantly associated with $21 \%$ reduced odds of BC (aOR: 0.79; 95\% CI: 0.67, 0.95 ) in adjusted models but was no longer significant in models additionally adjusting for height and weight (aOR: 0.75, 95\% CI: 0.15, 3.72). Weight $(\mathrm{kg})$ in the highest quartile was associated with $37 \%$ reduced odds of BC (aOR: 0.63, 95\% CI: 0.38, 1.04) in adjusted models, however this was not statistically significant, although each SD increase in weight was associated with significantly reduced odds of $\mathrm{BC}$ (aOR: 0.83; 95\% CI: 0.69, 0.98).
Increased height was not significantly associated with odds of BC in fully adjusted models or in weight and height adjusted models. In sub-group analyses stratified by menopausal status, BMI and weight were significantly associated with reduced odds of $\mathrm{BC}$ only among pre/ peri-menopausal women (Fig. 3).

In multinomial logistic regression models evaluating BC molecular subtypes compared with controls (Table 4), highest versus lowest quartiles of BMI was associated with reduced but not statistically significant odds of each $\mathrm{BC}$ subtype, although the association of each SD increase in BMI with reduced odds of Luminal B subtype was statistically significant (aOR: $0.63 ; 95 \%$ CI: 0.42 , $0.95)$. When we stratified by menopausal status, each unit SD increase in BMI was significantly associated with reduced odds of Luminal A (aOR 0.59; 95\% CI: 0.35, 0.99), Luminal B (aOR: 0.48; 95\% CI: 0.26, 0.90), and HER2-enriched (aOR: 0.49; 95\% CI: 0.27, 0.90) BC among pre/peri-menopausal women, and reduced odds of TNBC (aOR: 0.55; 95\% CI: 0.32, 0.95) among postmenopausal women. Height in the highest quartile was associated with $78 \%$ reduced odds of HER2-enriched BC (aOR: 0.22; 95\% CI: 0.06, 0.83). Additionally, each unit

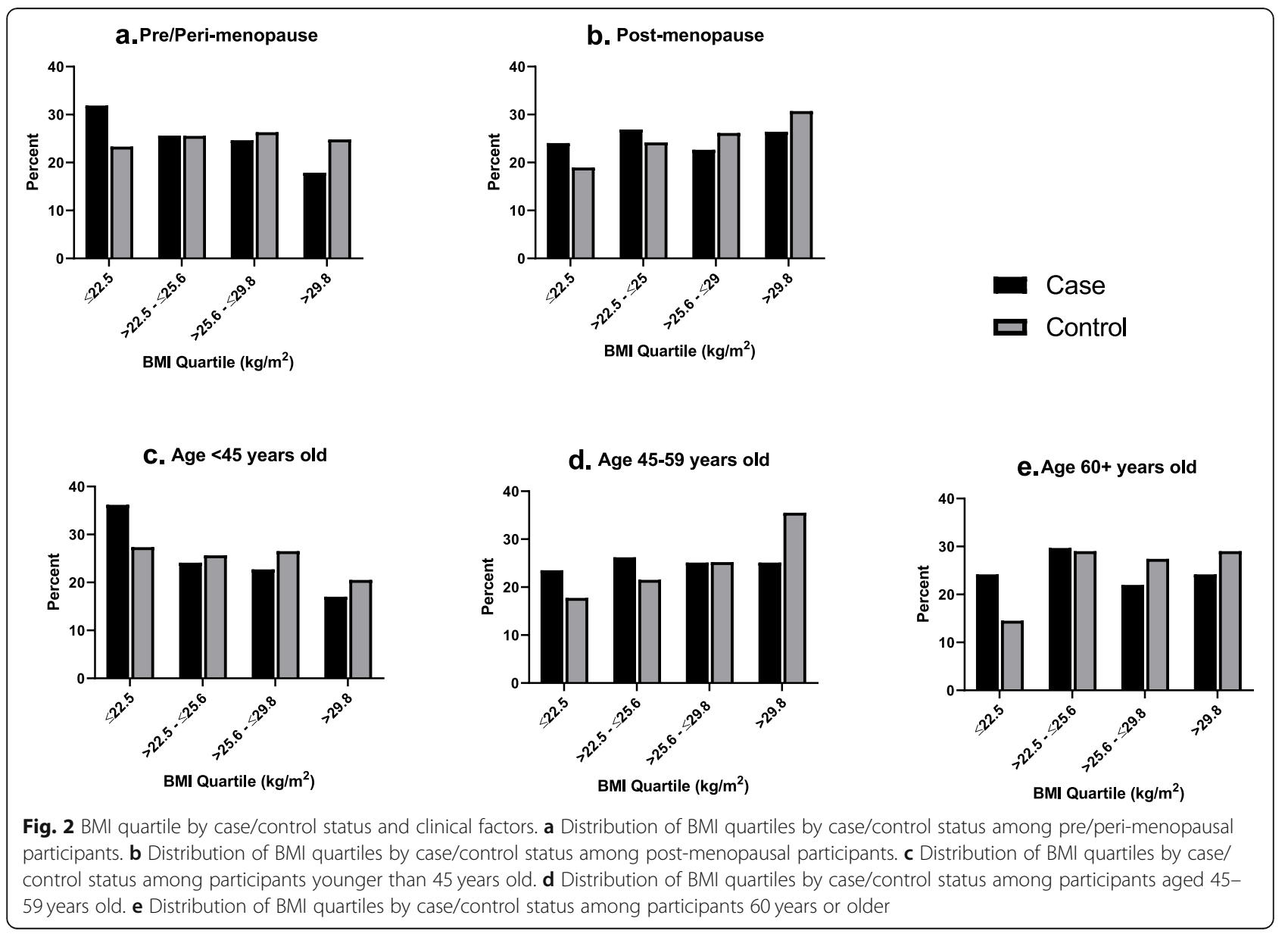


Table 3 Associations between body composition and breast cancer

\begin{tabular}{|c|c|c|c|c|}
\hline & $\begin{array}{l}\text { Model } 1^{\mathrm{a}} \\
\text { OR }(95 \% \mathrm{Cl})\end{array}$ & $\begin{array}{l}\text { Model } 2^{b} \\
\text { aOR }(95 \% \text { Cl) }\end{array}$ & $\begin{array}{l}\text { Model } 3^{\mathrm{c}} \\
\text { aOR }(95 \% \mathrm{Cl})\end{array}$ & $\begin{array}{l}\text { Model } 4^{\mathrm{d}} \\
\text { aOR }(95 \% \mathrm{Cl})\end{array}$ \\
\hline \multicolumn{5}{|l|}{ BMI $\left(\mathrm{kg} / \mathrm{m}^{2}\right)$ quartile } \\
\hline$\leq 22.5$ & Ref. & Ref. & Ref. & Ref. \\
\hline$>22.5-\leq 25.6$ & $0.79(0.52,1.22)$ & $0.76(0.49,1.18)$ & $0.73(0.44,1.22)$ & $0.60(0.31,1.19)$ \\
\hline$>25.6-\leq 29.8$ & $0.68(0.44,1.04)$ & $0.65(0.42,1.01)$ & $0.63(0.38,1.04)$ & $0.38(0.15,0.95)$ \\
\hline$>29.8$ & $0.60(0.39,0.92)$ & $0.56(0.36,0.86)$ & $0.56(0.33,0.92)$ & $0.32(0.10,1.09)$ \\
\hline P-trend & .01 & .01 & .02 & .06 \\
\hline aOR (95\% Cl) per unit SD & $0.81(0.70,0.94)$ & $0.79(0.68,0.93)$ & $0.79(0.67,0.95)$ & $0.75(0.15,3.72)$ \\
\hline \multicolumn{5}{|l|}{ Height $(\mathrm{cm})$ quartile } \\
\hline$\leq 156.0$ & Ref. & Ref. & Ref. & Ref. \\
\hline$>156.0-\leq 160.0$ & $1.05(0.67,1.64)$ & $1.07(0.68,1.68)$ & $1.05(0.62,1.76)$ & $0.90(0.51,1.57)$ \\
\hline$>160.0-\leq 164.0$ & $1.41(0.94,2.10)$ & $1.45(0.97,2.16)$ & $1.29(0.81,2.05)$ & $1.12(0.66,1.90)$ \\
\hline$>164.0$ & $1.22(0.81,1.84)$ & $1.27(0.84,1.91)$ & $1.08(0.67,1.73)$ & $0.87(0.48,1.60)$ \\
\hline P-trend & .17 & .13 & .55 & .85 \\
\hline aOR $(95 \% \mathrm{Cl})$ per unit SD & $1.15(0.99,1.34)$ & $1.16(1.00,1.36)$ & $1.07(0.90,1.28)$ & $1.05(0.56,1.96)$ \\
\hline \multicolumn{5}{|l|}{ Weight (kg) quartile } \\
\hline$\leq 56.7$ & Ref. & Ref. & Ref. & Ref. \\
\hline$>56.7-\leq 65.0$ & $0.85(0.56,1.31)$ & $0.84(0.54,1.28)$ & $0.75(0.46,1.24)$ & $1.09(0.55,2.15)$ \\
\hline$>65.0-\leq 77.0$ & $0.89(0.58,1.37)$ & $0.87(0.56,1.34)$ & $0.93(0.56,1.57)$ & $2.04(0.78,5.29)$ \\
\hline$>77.0$ & $0.74(0.48,1.14)$ & $0.71(0.46,1.10)$ & $0.63(0.38,1.04)$ & $1.70(0.48,5.98)$ \\
\hline P-trend & .22 & .16 & .14 & .31 \\
\hline aOR (95\% Cl) per unit SD & $0.86(0.74,1.00)$ & $0.85(0.73,0.99)$ & $0.83(0.69,0.98)$ & $1.06(0.20,5.75)$ \\
\hline
\end{tabular}

P-trend based on continuous predictors; aOR per unit SD modeled as a one-unit increase in standard deviation of the variable from its mean-centered value Bolded values indicate significance at $p<.05$

Abbreviations: $O R$ odds ratio, $C l$ confidence interval, $a O R$ adjusted odds ratio, $S D$ standard deviation

Logistic regression models predicted odds of breast cancer. ${ }^{a}$ Model 1, unadjusted; ${ }^{b}$ Model 2, adjusted for age; ${ }^{c}$ Model 3 , additionally adjusted for clinical and reproductive characteristics: age at menarche, number of pregnancies, number of births, menopausal status, and prior hypertension diagnosis; ${ }^{\mathrm{d} M o d e l} 4$, additionally adjusted for all body composition measures: BMI, height, and weight

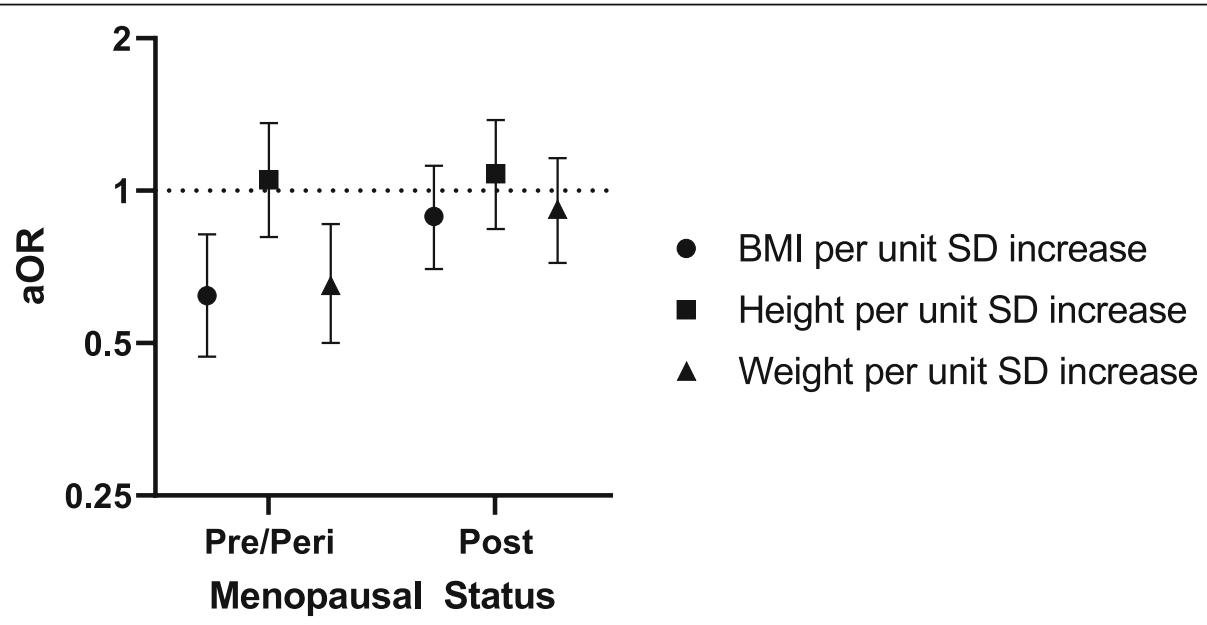

Fig. 3 Associations between body composition and breast cancer by menopausal status. Logistic regression models predicting odds of breast cancer. Adjusted for age, age at menarche, number of pregnancies, number of births, and prior hypertension diagnosis 
Table 4 Associations between body composition measures and breast cancer subtype

\begin{tabular}{|c|c|c|c|c|}
\hline Model 3 & $\begin{array}{l}\text { Luminal A } \\
\text { aOR }(95 \% \mathrm{Cl})\end{array}$ & $\begin{array}{l}\text { Luminal B } \\
\text { aOR }(95 \% \mathrm{Cl})\end{array}$ & $\begin{array}{l}\text { Triple Negative } \\
\text { aOR }(95 \% \mathrm{Cl})\end{array}$ & $\begin{array}{l}\text { HER2 } \\
\text { aOR }(95 \% \mathrm{Cl})\end{array}$ \\
\hline \multicolumn{5}{|l|}{ BMI $\left(\mathrm{kg} / \mathrm{m}^{2}\right)$ quartile } \\
\hline$\leq 22.5$ & Ref & Ref & Ref & Ref \\
\hline $22.5-25.5$ & $0.55(0.22,1.41)$ & $0.94(0.36,2.50)$ & $0.41(0.16,1.06)$ & $0.97(0.34,2.76)$ \\
\hline $25.6-29.8$ & $0.33(0.12,0.90)$ & $0.63(0.22,1.76)$ & $0.47(0.19,1.14)$ & $0.70(0.24,2.07)$ \\
\hline$>29.8$ & $0.51(0.21,1.24)$ & $0.35(0.11,1.13)$ & $0.48(0.20,1.13)$ & $0.68(0.23,1.97)$ \\
\hline P-trend & 0.10 & 0.06 & 0.13 & .38 \\
\hline aOR $(95 \%$ Cl) per unit SD & $0.82(0.59,1.16)$ & $0.63(0.42,0.95)$ & $0.81(0.58,1.12)$ & $0.74(0.50,1.09)$ \\
\hline Pre/peri-menopausal & $0.59(0.35,0.99)$ & $0.48(0.26,0.90)$ & $0.89(0.56,1.42)$ & $0.49(0.27,0.90)$ \\
\hline Post-menopausal & $1.03(0.64,1.68)$ & $0.66(0.36,1.21)$ & $0.55(0.32,0.95)$ & $1.10(0.61,1.98)$ \\
\hline \multicolumn{5}{|l|}{ Height (cm) quartile (all) } \\
\hline$\leq 156.0$ & Ref & Ref & Ref & Ref \\
\hline$>156.0-\leq 160.0$ & $0.88(0.29,2.68)$ & $0.76(0.24,2.41)$ & $1.33(0.53,3.36)$ & $0.83(0.31,2.22)$ \\
\hline$>160.0-\leq 164.0$ & $1.42(0.58,3.50)$ & $0.80(0.29,2.18)$ & $1.02(0.43,2.41)$ & $0.66(0.27,1.65)$ \\
\hline$>164.0$ & $1.39(0.55,3.51)$ & $1.18(0.46,3.03)$ & $1.01(0.41,2.45)$ & $0.22(0.06,0.83)$ \\
\hline P-trend & .35 & .74 & .89 & .03 \\
\hline aOR $(95 \%$ Cl) per unit SD & $1.20(0.86,1.67)$ & $1.09(0.76,1.57)$ & $1.05(0.77,1.44)$ & $0.74(0.51,1.07)$ \\
\hline Pre/peri-menopausal & $1.23(0.78,1.93)$ & $1.39(0.85,2.27)$ & $0.77(0.48,1.23)$ & $0.72(0.44,1.16)$ \\
\hline Post-menopausal & $1.19(0.71,2.01)$ & $0.78(0.46,1.33)$ & $1.43(0.89,2.28)$ & $0.78(0.42,1.44)$ \\
\hline \multicolumn{5}{|l|}{ Weight (kg) quartile (all) } \\
\hline$\leq 56.7$ & Ref & Ref & Ref & Ref \\
\hline$>56.7-\leq 65.0$ & $0.46(0.16,1.29)$ & $2.14(0.76,6.03)$ & $0.52(0.21,1.30)$ & $0.84(0.32,2.22)$ \\
\hline$>65.0-\leq 77.0$ & $0.65(0.25,1.73)$ & $1.32(0.42,4.18)$ & $0.77(0.32,1.88)$ & $0.48(0.15,1.54)$ \\
\hline$>77.0$ & $0.73(0.30,1.75)$ & $0.46(0.12,1.77)$ & $0.54(0.23,1.30)$ & $0.52(0.19,1.44)$ \\
\hline P-trend & .67 & .14 & .29 & .15 \\
\hline aOR $(95 \%$ Cl) per unit SD & $0.91(0.65,1.27)$ & $0.68(0.45,1.01)$ & $0.83(0.60,1.14)$ & $0.68(0.46,1.01)$ \\
\hline Pre/peri-menopausal & $0.70(0.43,1.16)$ & $0.59(0.33,1.06)$ & $0.81(0.50,1.30)$ & $0.45(0.24,0.84)$ \\
\hline Post-menopausal & $1.10(0.67,1.78)$ & $0.62(0.34,1.15)$ & $0.68(0.41,1.13)$ & $0.99(0.55,1.78)$ \\
\hline
\end{tabular}

Multinomial logistic regression models predicting odds of breast cancer subtype versus controls. Adjusted for reproductive and clinical characteristics: age, age at menarche, number of pregnancies, number of births, menopausal status (not included in stratified models), and prior hypertension diagnosis P-trend based on continuous predictors

aOR per unit SD was modeled as a one-unit increase in standard deviation of the body composition variable from its mean-centered value. These models were stratified by menopausal status

Bolded values indicate significance at $p<.05$

Abbreviations: $a O R$ adjusted odds ratio, $C l$ confidence interval, SD standard deviation

SD increase in weight was significantly associated with $55 \%$ reduced odds of HER2-enriched $\mathrm{BC}$ (aOR: 0.45; 95\% CI: $0.24,0.84$ ) only among pre/per-menopausal women.

\section{Discussion}

In the first contemporary cohort of newly diagnosed African $\mathrm{BC}$ cases and healthy controls, increasing BMI and weight were significantly associated with reduced odds of $\mathrm{BC}$ overall, but no statistically significant association was noted with respect to height. Similar associations were observed among both pre/peri-menopausal and post-menopausal women, but only reached statistical significance among pre/peri-menopausal women. In our analysis of $\mathrm{BC}$ subtypes, higher BMI was significantly associated with reduced odds of Luminal A, Luminal B, and HER2-enriched BC among pre/peri-menopausal women, and reduced odds of TNBC among postmenopausal women.

Previous studies have evaluated the association between measures of body composition and $\mathrm{BC}$ in various patient populations, with results suggesting a positive association of BMI with $\mathrm{BC}$ risk among post-menopausal women, and inverse association among pre-menopausal women [10-14]. This is consistent with our findings of significant inverse associations with BMI and weight for 
pre-menopausal women. A few studies have evaluated these associations among Nigerian women, although most were published over a decade ago prior to the accelerated increase in obesity rates in the region. Specifically, two separate studies observed that a higher waistto-hip ratio was associated with significantly increased risk of $\mathrm{BC}[17,18]$, and three studies showed that height was associated with significantly increased risk of $\mathrm{BC}$ $[16,17,23]$. Additionally, obesity (defined as BMI $\geq 30$ ) was found to be associated with increased odds of $\mathrm{BC}$, although the results were not statistically significant overall or among pre- or post-menopausal women [16].

For the first time, we present results on the association between measures of body composition and BC subtypes compared to controls among West African women. We found reduced odds of Luminal A, Luminal B, and HER2-enriched BC among pre/peri-menopausal women, and reduced odds of TNBC among post-menopausal women with increased BMI. Prior studies on this topic are conflicting, and suggest that the associations between body composition and $\mathrm{BC}$ subtypes vary by menopausal status. A case-control study among Black and White women in the United States documented a positive association between elevated waist-to-hip ratio and Luminal A BC among post-menopausal women [25]. Another analysis, which included studies in the Breast Cancer Association Consortium, a primarily European population, found that elevated BMI in younger women was more strongly associated with hormone receptor positive tumors (Luminal subtypes) [32], and another study from the United States noted that among post-menopausal women, BMI was associated with an increased odds of $\mathrm{ER}+\mathrm{PR}+\mathrm{BC}$ [33]. In contrast, a meta-analysis of studies from the US, Europe, and Asia found that ER+/PR+ tumors were less likely to develop among higher weight pre-menopausal women, but more likely to develop among higher weight post-menopausal women [34]. Our findings are consistent with a prior study that found an inverse association between BMI and risk of TNBC [35]. However, our findings are inconsistent with other past studies showing positive associations between obesity and TNBC [24, 25,36], most of which were conducted in study populations from the United States, Europe, and Asia, and may not be representative of the $\mathrm{BC}$ phenotypes observed in Nigeria.

Despite the documented inverse association between $\mathrm{BMI}$ and $\mathrm{BC}$ risk among pre-menopausal women, the underlying biological mechanisms remain an active area of research. For instance, lower levels of progesterone among pre-menopausal obese women may lead to a lower risk of $\mathrm{BC}$ because progesterone is thought to have a primarily pro-proliferative role in the adult breast [37]. However, further research is needed to better explain how excess adiposity contributes to BC risk across different subtypes. While our findings may suggest lower $\mathrm{BC}$ risk among obese patients relative to lean patients, it is well-established that $\mathrm{BC}$ survival is negatively impacted by obesity [38]. These seemingly paradoxical observations, dubbed the obesity paradox, may be a consequence of differences in treatment response among differently weighted patients [39]. Additionally, biological mechanisms that explain the higher risk of TNBC in women of West African descent are not wellunderstood. We urge additional research among African populations to elucidate population-specific risk factors that may contribute to these outcomes.

Several considerations are relevant for our study. Given the predominance of the aggressive phenotype (high grade and TN subtype) of $\mathrm{BC}$ in Nigeria, also reflected in the current study population, it is possible for cases to have experienced disease associated weight loss prior to recruitment. It is worth noting that TNBCs grow quickly and are more likely to be clinically apparent compared with $\mathrm{ER}+/ \mathrm{PR}+$ cancers, which are more likely to be detected by screening mammography [40]. The low prevalence of mammogram screening in Nigeria [41], reflected in our sample with less than $19 \%$ of cases having had a previous mammogram screening, may contribute to the high prevalence of TNBC. However, it is important to note that studies among African American women, with similar BC morphological features to Nigerian women [9], have been inconsistent, with some suggesting positive associations between excess adiposity and $\mathrm{BC}$ risk $[42,43]$, while others have reported no significant associations $[44,45]$. The exact relationship between $\mathrm{BMI}$ and $\mathrm{BC}$ risk remains conflicting for women of African descent, indicating a need for further study in a larger cohort, especially given the context in which $\mathrm{BC}$ disease is distinctly more aggressive [6-8].

There are several strengths and limitations of this study relevant to the interpretation of these results. Since covariate data were self-reported at diagnosis, we cannot exclude the possibility of recall bias. However, our main exposures of interest, measures of excess adiposity, were obtained consistently across study participants by trained study personnel. Furthermore, because BMI, height, and weight were recorded at the time of diagnosis, we are unable to rule out the possibility of reverse causality. Additionally, we note the limitation of relying purely on BMI (height and weight) as a measure of excess adiposity. Individuals with the same BMI can have different body fat distributions [46]. Nevertheless, our study has important strengths, including the use of histologically confirmed cases of $\mathrm{BC}$ from multiple tertiary institutions in Nigeria where the majority of cancer patients receive treatment, and a vital contribution of diverse patient populations to the $\mathrm{BC}$ literature. Our study is the first to characterize the association between 
measures of body composition and $\mathrm{BC}$ risk among West African women by molecular subtype.

In conclusion, measures of body composition were associated with $\mathrm{BC}$ and these associations varied by molecular subtypes. Larger studies of women of African descent are needed to better characterize these associations and enhance population-specific $\mathrm{BC}$ prevention strategies.

\section{Abbreviations}

BMl: Body mass index; BC: Breast cancer; CKD: Chronic kidney disease; ER: Estrogen receptor; HER2: Human epidermal growth factor-2; H3A: Human Heredity and Health Africa; IHC: Immunohistochemistry; MEND: Mechanisms for Established and Novel Risk Factors for Breast Cancer in Women of Nigerian Descent; PR: Progesterone receptor (PR); SD: Standard deviation; TN: Triple-negative

\section{Acknowledgements}

We acknowledge the role of the H3Africa Consortium in making this research possible though the sharing of data. The National Institutes of Health (USA) and Wellcome Trust (UK) have provided the core funding for the H3Africa Consortium. We thank the many MEND investigators who contributed substantially to the inception and design of the study, and the patients and families who participated in the MEND study for their vital contribution in advancing the science of cancer in Nigeria and globally. We acknowledge the important contribution of the MEND research nurses: Cordelia Ibeneme, Peju Olabanji, Rebecca Israel, Esther Akinwale, Deborah Awodeyi, and Shukurat Oduola.

H3 Africa Kidney Research Network

Samuel Ajayi ${ }^{14}$

Yemi Raji ${ }^{14}$

Timothy Olanrewaju ${ }^{15}$

Charlotte Osafo $^{16}$

Ifeoma Ulasi ${ }^{17}$

Adanze Asinobi ${ }^{14}$

Cheryl A. Winkler ${ }^{18}$

David Burke ${ }^{19}$

Fatiu Arogundade 20

Ivy Ekem ${ }^{21}$

Jacob Plange-Rhule 22*** $^{\text {2 }}$

Manmak Mamven ${ }^{23}$

Michael Mate-kole ${ }^{16}$

Olukemi Amodu ${ }^{14}$

Richard Cooper ${ }^{24}$

Sampson Antwi ${ }^{22}$

Adebowale Adeyemo ${ }^{25}$

Titilayo $\|^{2}{ }^{26}$

Victoria Adabayeri ${ }^{16}$

Alexander Nyarko ${ }^{27}$

Anita Ghansah ${ }^{27}$

Ernestine Kubi Amos-Abanyie ${ }^{27}$

Priscilla Abena Akyaw ${ }^{27}$

Paul L. Kimmel ${ }^{28}$

Babatunde L. Salako ${ }^{14}$

Rulan S. Parekh ${ }^{29}$

Bamidele Tayo ${ }^{24}$

Rasheed Gbadegesin ${ }^{30}$

Michael Boehnke

Robert Lyons ${ }^{19}$

Frank (Chip) Brosius ${ }^{19}$

Daniel Clauw ${ }^{19}$

Chijioke Adindu ${ }^{15}$

Clement Bewaji ${ }^{15}$

Elliot Koranteng Tannor ${ }^{22}$

Perditer Okyere 22

Chuba ljoma $^{17}$

Nicki Tiffin ${ }^{31}$

Junaid Gamiedien ${ }^{31}$

Friedhelm Hildebrandt ${ }^{32}$
Charles Odenigbo ${ }^{33}$

Nonyelun Jisieike-Onuigbo ${ }^{33}$

Ifeoma Modebe ${ }^{33}$

Aliyu Abdu ${ }^{34}$

Patience Obiagwu ${ }^{34}$

Ogochukwu Okoye ${ }^{35}$

Adaobi Solarin ${ }^{36}$

Toyin Amira ${ }^{6}$

Christopher Esezobor ${ }^{6}$

Muhammad Makusidi ${ }^{37}$

Santosh Saraf ${ }^{38}$

Victor Gordeuk ${ }^{38}$

Gloria Ashuntangtang 39

Georgette Guenkam ${ }^{39}$

Folefack Kazi ${ }^{39}$

Olanrewaju Adedoyin ${ }^{15}$

Mignon McCullough ${ }^{40}$

Peter Nourse ${ }^{40}$

Uche Okafor ${ }^{17}$

Emmanuel Anigilaje ${ }^{23}$

Patrick Ikpebe ${ }^{36}$

Tola Odetunde ${ }^{17}$

Ngozi Mbanefo ${ }^{17}$

Wasiu Olowu ${ }^{20}$

Paulina Tindana ${ }^{16}$

Olubenga Awobusuyij ${ }^{36}$

Olugbenga Ogedegbe ${ }^{41}$

Opeyemi Olabisi42

Karl Skorecki ${ }^{43}$

Ademola Adebowale ${ }^{14}$

Matthias Kretzler ${ }^{19}$

Jeffrey Hodgin ${ }^{19}$

Dwomoa Adu ${ }^{16}$

Akinlolu Ojo ${ }^{44}$

Vincent Boima ${ }^{16}$

Affiliations

${ }^{14}$ Department of Medicine, Pediatrics and Institute of Child Health, University

of Ibadan, Ibadan, Nigeria

${ }^{15}$ University of Ilorin, Ilorin, Nigeria

${ }^{16}$ University of Ghana Medical School, Accra, Ghana

${ }^{17}$ University of Nigeria, Enugu State, Nigeria

${ }^{18}$ Basic Research Laboratory, Frederick National Laboratory for Cancer

Research, National Cancer Institute, Frederick, Maryland, USA

${ }^{19}$ Departments of Human Genetics, Internal Medicine and Pathology,

University of Michigan, Ann Arbor, Michigan, USA

${ }^{20}$ Obafemi Awolowo University, lle- Ife, Nigeria

${ }^{21}$ University of Cape Coast, Cape Coast, Ghana

${ }^{22}$ Kwame Nkrumah University of Science and Technology, Kumasi, Ghana

${ }^{23}$ University of Abuja, Abuja, Nigeria

${ }^{24}$ Parkinson School of Health Sciences and Public Health, Loyola University,

Chicago, Illinois, USA

${ }^{25}$ Centre for Research on Genomics and Global Health, National Human

Genome Research Institute, National Institutes of Health, Bethesda, Maryland, USA

${ }^{26}$ Division of Nephrology, Boston Medical Center, Boston University School of Medicine, Boston, Massachusetts, USA

${ }^{27}$ Noguchi Memorial Institute for Medical Research, University of Ghana,

Ghana

${ }^{28}$ National Institute of Diabetes and Digestive and Kidney Disease, Bethesda,

Maryland, USA

${ }^{29}$ Department of Pediatrics, University of Toronto, Toronto, Canada

${ }^{30}$ Department of Pediatrics, Duke University Medical Center, Durham, North Carolina, USA

${ }^{31}$ University of Western Cape, Cape Town, South Africa

${ }^{32}$ Harvard Medical School, Harvard University, Boston, Massachusetts, USA

${ }^{33}$ Nnamdi Azikiwe University Teaching Hospital, Nnewi, Nigeria

${ }^{34}$ Aminu Kano Teaching Hospital, Kano, Nigeria

${ }^{35}$ Delta State University Teaching Hospital, Warri, Nigeria

${ }^{36}$ Lagos State University Teaching Hospital, Lagos, Nigeria

${ }^{37}$ Usmanu Danfodiyo University Teaching Hospital, Sokoto, Nigeria

${ }^{38}$ University of Illinois at Chicago, Chicago Illinois, USA 
${ }^{39}$ University of Yaoundé, Yaoundé, Cameroon

${ }^{40}$ University of Cape Town, Cape Town, South Africa

${ }^{41}$ New York University, New York City, New York, USA

${ }^{42}$ Duke University, Durham, North Carolina, USA

${ }^{43}$ Technion-Israel Institute of Technology, Haifa, Israel

${ }^{44}$ University of Kansas School of Medicine, Kansas City, Kansas, USA

**Deceased

\section{Authors' contributions}

Conceptualization, T.A.; methodology, T.A.; formal analysis, T.A., K.J., A.G., T.O.; resources, T.A., H3.A.K.R.N.; writing-original draft preparation, T.A., A.G.; writing-review and editing, T.A., K.J., A.G., T.O., V.S., A.D., O.S., A.H., O.A., G.O., A.A., O.A., T.O., O.O., O.A., A.A., O.A., A.O., A.A., T.O.T., D.A., A.D.; funding acquisition, T.A. All authors have read and agreed to the final version of the manuscript.

\section{Funding}

This research was specifically funded by National Institutes of Health, National Cancer Institute, Fogarty International Center (K01TW010271, T.A.). The views expressed in this paper do not represent the views of the National Institutes of Health, H3Africa Consortium or their funders.

\section{Availability of data and materials}

The datasets used and/or analyzed during the current study are available from the corresponding author on reasonable request.

\section{Declarations}

\section{Ethics approval and consent to participate}

All procedures performed in studies involving human participants were in accordance with the ethical standards of the institutional and/or national research committee and with the 1964 Helsinki declaration and its later amendments or comparable ethical standards. The study was approved by the Institutional Review Board of Duke University (Pro00102004). This article does not contain any studies with animals performed by any of the authors. Informed consent was obtained from all individual participants included in the study.

\section{Consent for publication}

Not applicable.

\section{Competing interests}

The authors declare that they have no competing interests.

\section{Author details}

${ }^{1}$ Department of Population Health Sciences, School of Medicine, Duke University, Durham, NC, USA. ²Duke Cancer Institute, School of Medicine, Duke University, Durham, NC, USA. ${ }^{3}$ Duke Global Health Institute, Duke University, Durham, NC, USA. ${ }^{4}$ Trinity College of Arts and Sciences, Duke University, Durham, NC, USA. ${ }^{5}$ Department of Epidemiology, University of North Carolina Gillings School of Global Public Health, Chapel Hill, NC, USA. ${ }^{6}$ College of Medicine \& Lagos University Teaching Hospital, University of Lagos, Lagos, Lagos State, Nigeria. 'Department of Pathology, School of Medicine, Duke University, Durham, NC, USA. ${ }^{8}$ Obafemi Awolowo University Teaching Hospital, Ile-Ife, Osun State, Nigeria. 'University College Hospital, University of Ibadan, Ibadan, Oyo State, Nigeria. ${ }^{10}$ Federal Medical Center, Abeokuta, Ogun State, Nigeria. ${ }^{11}$ Our Lady of Apostle Catholic Hospital, Ibadan, Oyo State, Nigeria. ${ }^{12}$ University of Alabama at Birmingham, Birmingham, AL, USA. '13 University of Kentucky, Lexington, KY, USA.

${ }^{14}$ University of Kansas Medical Center, Kansas City, KS, USA.

Received: 17 April 2021 Accepted: 31 August 2021

Published online: 25 September 2021

\section{References}

1. Bray F, Ferlay J, Soerjomataram I, Siegel RL, Torre LA, Jemal A. Global cancer statistics 2018: GLOBOCAN estimates of incidence and mortality worldwide for 36 cancers in 185 countries. CA Cancer J Clin. 2018;68(6):394-424. https://doi.org/10.3322/caac.21492.

2. Azubuike SO, Muirhead C, Hayes L, McNally R. Rising global burden of breast cancer: the case of sub-Saharan Africa (with emphasis on Nigeria) and implications for regional development: a review. World J Surg Oncol. 2018;16(1):63. https://doi.org/10.1186/s12957-018-1345-2.

3. Adeloye D, Sowunmi OY, Jacobs W, David RA, Adeosun AA, Amuta AO, et al. Estimating the incidence of breast cancer in Africa: a systematic review and meta-analysis. J Glob Health. 2018;8(1):010419. https://doi.org/10. 7189/jogh.08.010419.

4. Mckeown RE. The epidemiologic transition: changing patterns of mortality and population dynamics. Am J Lifestyle Med. 2009;3(1 Suppl):19s-26s.

5. Fitzmaurice C, Allen C, Barber RM, Barregard L, Bhutta ZA, Brenner $\mathrm{H}$, et al. Global, regional, and national cancer incidence, mortality, years of life lost, years lived with disability, and disability-adjusted life-years for 32 cancer groups, 1990 to 2015: a systematic analysis for the global burden of disease study. JAMA Oncol. 2017;3(4):524-48. https://doi.org/10.1001/jamaoncol.201 6.5688.

6. Pitt JJ, Riester M, Zheng Y, Yoshimatsu TF, Sanni A, Oluwasola O, et al. Characterization of Nigerian breast cancer reveals prevalent homologous recombination deficiency and aggressive molecular features. Nat Commun. 2018;9(1):4181. https://doi.org/10.1038/s41467-018-06616-0.

7. Huo D, Ikpatt F, Khramtsov A, Dangou JM, Nanda R, Dignam J, et al. Population differences in breast cancer: survey in indigenous African women reveals over-representation of triple-negative breast cancer. J Clin Oncol. 2009;27(27):4515-21. https://doi.org/10.1200/JCO.2008.19.6873.

8. Wright N, Rida P, Rakha E, Agboola A, Aneja R. Panoptic overview of triplenegative breast cancer in Nigeria: current challenges and promising global initiatives. J Glob Oncol. 2018;4:1-20. https://doi.org/10.1200/JG0.17.00116.

9. Newman LA, Kaljee LM. Health disparities and triple-negative breast cancer in African American women: a review. JAMA Surg. 2017;152(5):485-93. https://doi.org/10.1001/jamasurg.2017.0005.

10. Renehan AG, Tyson M, Egger M, Heller RF, Zwahlen M. Body-mass index and incidence of cancer: a systematic review and meta-analysis of prospective observational studies. Lancet (London, England). 2008;371(9612): 569-78.

11. Schoemaker MJ, Nichols HB, Wright LB, Brook MN, Jones ME, O'Brien KM, et al. Association of body mass index and age with subsequent breast cancer risk in premenopausal women. JAMA Oncol. 2018;4(11):e181771. https://doi.org/10.1001/jamaoncol.2018.1771.

12. Al-Ajmi K, Lophatananon A, Ollier W, Muir KR. Risk of breast cancer in the UK biobank female cohort and its relationship to anthropometric and reproductive factors. PLoS One. 2018;13(7):e0201097. https://doi.org/10.13 71/journal.pone.0201097.

13. His M, Biessy C, Torres-Mejía G, Ángeles-Llerenas A, Alvarado-Cabrero I, Sánchez Gl, et al. Anthropometry, body shape in early-life and risk of premenopausal breast cancer among Latin American women: results from the PRECAMA study. Sci Rep. 2020;10(1):2294. https://doi.org/10.1038/s41 598-020-59056-6.

14. Suzuki Y, Tsunoda H, Kimura T, Yamauchi H. BMI change and abdominal circumference are risk factors for breast cancer, even in Asian women. Breast Cancer Res Treat. 2017;166(3):919-25. https://doi.org/10.1007/s10549017-4481-4.

15. Chukwuonye II, Chuku A, John C, Ohagwu KA, Imoh ME, Isa SE, et al. Prevalence of overweight and obesity in adult Nigerians - a systematic review. Diabetes Metab Syndr Obes. 2013;6:43-7. https://doi.org/10.2147/ DMSO.S38626.

16. Adebamowo CA, Ogundiran TO, Adenipekun AA, Oyesegun RA, Campbell $\mathrm{OB}$, Akang EU, et al. Obesity and height in urban Nigerian women with breast cancer. Ann Epidemiol. 2003;13(6):455-61. https://doi.org/10.1016/S1 047-2797(02)00426-X.

17. Okobia MN, Bunker CH, Zmuda JM, Osime U, Ezeome ER, Anyanwu SN, et al. Anthropometry and breast cancer risk in Nigerian women. Breast J. 2006;12(5):462-6. https://doi.org/10.1111/j.1075-122X.2006.00304.x.

18. Ogundiran TO, Huo D, Adenipekun A, Campbell O, Oyesegun R, Akang E, et al. Body fat distribution and breast cancer risk: findings from the Nigerian breast cancer study. Cancer Causes Control. 2012;23(4):565-74. https://doi. org/10.1007/s10552-012-9916-y.

19. Saklayen MG. The global epidemic of the metabolic syndrome. Curr Hypertens Rep. 2018;20(2):12. https://doi.org/10.1007/s11906-018-0812-z.

20. Dibaba DT, Braithwaite D, Akinyemiju T. Metabolic syndrome and the risk of breast cancer and subtypes by race, menopause and BMI. Cancers. 2018; 10(9):299.

21. Qian F, Wang S, Mitchell J, McGuffog L, Barrowdale D, Leslie G, et al. Height and body mass index as modifiers of breast cancer risk in BRCA1/2 
mutation carriers: a mendelian randomization study. J Natl Cancer Inst. 2019;111(4):350-64. https://doi.org/10.1093/jnci/djy132.

22. Zhang B, Shu XO, Delahanty RJ, Zeng C, Michailidou K, Bolla MK, et al. Height and breast cancer risk: evidence from prospective studies and mendelian randomization. J Natl Cancer Inst. 2015;107(11):djv219.

23. Ogundiran TO, Huo D, Adenipekun A, Campbell O, Oyesegun R, Akang E, et al. Case-control study of body size and breast cancer risk in Nigerian women. Am J Epidemiol. 2010;172(6):682-90. https://doi.org/10.1093/aje/ kwq180.

24. Gershuni V, Li YR, Williams AD, So A, Steel L, Carrigan E, et al. Breast cancer subtype distribution is different in normal weight, overweight, and obese women. Breast Cancer Res Treat. 2017;163(2):375-81. https://doi.org/10.1 007/s10549-017-4192-x.

25. Millikan RC, Newman B, Tse CK, Moorman PG, Conway K, Dressler LG, et al. Epidemiology of basal-like breast cancer. Breast Cancer Res Treat. 2008; 109(1):123-39. https://doi.org/10.1007/s10549-007-9632-6.

26. Akinyemiju T, Salako O, Daramola A, Alatise O, Adeniyi A, Ogun G, et al. Collaborative molecular epidemiology study of metabolic dysregulation, DNA methylation, and breast cancer risk among Nigerian women: MEND study objectives and design. J Glob Oncol. 2019;5:1-9. https://doi.org/10.12 00/JGO.18.00226.

27. Osafo C, Raji YR, Burke D, Tayo BO, Tiffin N, Moxey-Mims MM, et al. Human heredity and health (H3) in Africa kidney disease research network: a focus on methods in sub-Saharan Africa. Clin J Am Soc Nephrol. 2015;10(12): 2279-87. https://doi.org/10.2215/CJN.11951214.

28. Mukherjee T. Interpretation of ER and Her2neu hormonal receptor in breast cancer. Med J Armed Forces India. 2016;72(1):99.

29. Hammond ME, Hayes DF, Dowsett M, Allred DC, Hagerty KL, Badve S, et al American Society of Clinical Oncology/College of American Pathologists guideline recommendations for immunohistochemical testing of estrogen and progesterone receptors in breast cancer. J Clin Oncol. 2010;28(16): 2784-95. https://doi.org/10.1200/JCO.2009.25.6529.

30. Wolff AC, Hammond ME, Schwartz JN, Hagerty KL, Allred DC, Cote RJ, et al. American Society of Clinical Oncology/College of American Pathologists guideline recommendations for human epidermal growth factor receptor 2 testing in breast cancer. Arch Pathol Lab Med. 2007;131(1):18-43. https:// doi.org/10.5858/2007-131-18-ASOCCO.

31. Jackson AS, Ellis KJ, McFarlin BK, Sailors MH, Bray MS. Body mass index bias in defining obesity of diverse young adults: the Training Intervention and Genetics of Exercise Response (TIGER) study. Br J Nutr. 2009;102(7):1084-90. https://doi.org/10.1017/S0007114509325738.

32. Yang XR, Chang-Claude J, Goode EL, Couch FJ, Nevanlinna H, Milne RL, et al. Associations of breast cancer risk factors with tumor subtypes: a pooled analysis from the breast cancer association consortium studies. J Natl Cancer Inst. 2011;103(3):250-63. https://doi.org/10.1093/jnci/djq526.

33. Enger SM, Ross RK, Paganini-Hill A, Carpenter CL, Bernstein L. Body size, physical activity, and breast cancer hormone receptor status: results from two case-control studies. Cancer Epidemiol Biomarkers Prev. 2000;9(7):6817.

34. Suzuki R, Orsini N, Saji S, Key TJ, Wolk A. Body weight and incidence of breast cancer defined by estrogen and progesterone receptor status--a meta-analysis. Int J Cancer. 2009;124(3):698-712. https://doi.org/10.1002/ ijc.23943.

35. Nagrani R, Mhatre S, Rajaraman P, Soerjomataram I, Boffetta P, Gupta S, et al. Central obesity increases risk of breast cancer irrespective of menopausal and hormonal receptor status in women of South Asian Ethnicity. Eur J Cancer. 2016;66:153-61.

36. Agresti R, Meneghini E, Baili P, Minicozzi P, Turco A, Cavallo I, et al. Association of adiposity, dysmetabolisms, and inflammation with aggressive breast cancer subtypes: a cross-sectional study. Breast Cancer Res Treat. 2016;157(1):179-89. https://doi.org/10.1007/s10549-016-3802-3.

37. Dowsett M, Folkerd E. Reduced progesterone levels explain the reduced risk of breast cancer in obese premenopausal women: a new hypothesis. Breast Cancer Res Treat. 2015;149(1):1-4. https://doi.org/10.1007/s10549014-3211-4.

38. Chan DS, Vieira AR, Aune D, Bandera EV, Greenwood DC, McTiernan A, et al. Body mass index and survival in women with breast cancer-systematic literature review and meta-analysis of 82 follow-up studies. Ann Oncol. 2014;25(10):1901-14. https://doi.org/10.1093/annonc/mdu042.
39. Lennon H, Sperrin M, Badrick E, Renehan AG. The obesity paradox in cancer: a review. Curr Oncol Rep. 2016;18(9):56. https://doi.org/10.1007/s11912-0160539-4.

40. Krizmanich-Conniff KM, Paramagul C, Patterson SK, Helvie MA, Roubidoux MA, Myles JD, et al. Triple receptor-negative breast cancer: imaging and clinical characteristics. AJR Am J Roentgenol. 2012;199(2):458-64. https://doi. org/10.2214/AJR.10.6096.

41. Bello TO, Olugbenga-Bello Al, Oguntola AS, Adeoti ML, Ojemakinde OM. Knowledge and practice of breast cancer screening among female nurses and lay women in Osogbo, Nigeria. West Afr J Med. 2011;30(4):296-300.

42. Schatzkin A, Palmer JR, Rosenberg L, Helmrich SP, Miller DR, Kaufman DW, et al. Risk factors for breast cancer in black women. J Natl Cancer Inst. 1987; 78(2):213-7.

43. Zhu K, Caulfield J, Hunter S, Roland CL, Payne-Wilks K, Texter L. Body mass index and breast cancer risk in African American women. Ann Epidemiol. 2005;15(2):123-8. https://doi.org/10.1016/j.annepidem.2004.05.011.

44. Adams-Campbell LL, Kim KS, Dunston G, Laing AE, Bonney G, Demenais F. The relationship of body mass index to reproductive factors in pre- and postmenopausal African-American women with and without breast cancer. Obes Res. 1996;4(5):451-6. https://doi.org/10.1002/j.1550-8528.1996.tb00253. $x$.

45. Palmer JR, Adams-Campbell LL, Boggs DA, Wise LA, Rosenberg L. A prospective study of body size and breast cancer in black women. Cancer Epidemiol Biomarkers Prev. 2007;16(9):1795-802. https://doi.org/10.1158/1 055-9965.EPI-07-0336.

46. Flegal KM, Shepherd JA, Looker AC, Graubard Bl, Borrud LG, Ogden CL, et al. Comparisons of percentage body fat, body mass index, waist circumference, and waist-stature ratio in adults. Am J Clin Nutr. 2009;89(2):500-8. https:// doi.org/10.3945/ajcn.2008.26847.

\section{Publisher's Note}

Springer Nature remains neutral with regard to jurisdictional claims in published maps and institutional affiliations.
Ready to submit your research? Choose BMC and benefit from:

- fast, convenient online submission

- thorough peer review by experienced researchers in your field

- rapid publication on acceptance

- support for research data, including large and complex data types

- gold Open Access which fosters wider collaboration and increased citations

- maximum visibility for your research: over $100 \mathrm{M}$ website views per year

At $\mathrm{BMC}$, research is always in progress.

Learn more biomedcentral.com/submissions 